\title{
Evidence for the start of planet formation in a young cir- cumstellar disk
}

Daniel Harsono ${ }^{1 *}$, Per Bjerkeli ${ }^{2,3}$, Matthijs H. D. van der Wiel ${ }^{4}$, Jon P. Ramsey $^{3}$, Luke T. Maud $^{1}$, Lars E. Kristensen ${ }^{3}$, Jes K. Jørgensen ${ }^{3}$

${ }^{1}$ Leiden Observatory, Leiden University, P.O. box 9513, 2300 RA Leiden, The Netherlands

${ }^{2}$ Department of Space, Earth and Environment, Chalmers University of Technology, Onsala Space Observatory, 43992 Onsala, Sweden

${ }^{3}$ Centre for Star and Planet Formation, Niels Bohr Institute \& Natural History Museum of Denmark, University of Copenhagen, Øster Voldgade 5-7, 1350 Copenhagen K, Denmark

${ }^{4}$ ASTRON, the Netherlands Institute for Radio Astronomy, P.O. Box 2, 7990 AA Dwingeloo, The Netherlands

The growth of dust grains in protoplanetary disks is a necessary first step towards planet formation ${ }^{1}$. This growth has been inferred via observations of thermal dust emission ${ }^{2}$ towards mature protoplanetary systems (age $>2$ million years) with masses that are, on average, similar to Neptune ${ }^{3}$. In contrast, the majority of confirmed exoplanets are heavier than Neptune ${ }^{4}$. Given that young protoplanetary disks are more massive than their mature counterparts, this suggests that planet formation starts early, but evidence for grain growth that is spatially and temporally coincident with a massive reservoir in young disks remains scarce. Here, we report observations on a lack of emission of carbon monoxide isotopologues within the inner $\sim 15$ au of a very young (age $\sim 100,000$ years) disk around the Solar-type 
protostar TMC1A. By using the absence of spatially resolved molecular line emission to infer the gas and dust content of the disk, we conclude that shielding by millimetre-size grains is responsible for the lack of emission. This suggests that grain growth and millimetre-size dust grains can be spatially and temporally coincident with a mass reservoir sufficient for giant planet formation. Hence, planet formation starts during the earliest, embedded phases in the life of young stars.

TMC1A (IRAS $04365+2535)$ is a young, low-mass $\left(\sim 1 M_{\text {Sun }}\right)$ protostellar system with a $100 \mathrm{au}$, rotationally supported disk in the nearby Taurus star-forming region ${ }^{5,6}$. Based on multiwavelength continuum observations, TMC1A has been classified as a Class I protostar ${ }^{7}$, implying that the protostar and disk system is surrounded by a substantial infalling circumstellar envelope from which it is still accreting material ${ }^{6,8}$. Using the Atacama Large Millimetre/sub-millimetre Array (ALMA), thermal dust continuum emission was observed at a wavelength of $1.3 \mathrm{~mm}$ along with the $J=2-1$ rotational transition of the carbon monoxide isotopologues ${ }^{13} \mathrm{CO}$ and $\mathrm{C}^{18} \mathrm{O}$. The disk in TMC1A is driving a wind ${ }^{9}$ and large-scale outflow ${ }^{6,10}$ which are carving a cavity in the envelope. Our observations spatially resolve the inner regions of the disk with an angular resolution of $0.06^{\prime \prime} \times 0.05^{\prime \prime}\left(\mathrm{PA}=25^{\circ}\right)$, corresponding to a spatial scale of 8.4 au $\times 7.0$ au when adopting a distance of 140 parsecs to the Taurus Molecular Cloud.

Previously, we reported resolved observations of a disk wind as revealed by ${ }^{12} \mathrm{CO}$ molecular emission in this system ${ }^{9}$. In this letter, we instead focus on the carbon monoxide isotopologue $\left({ }^{13} \mathrm{CO}\right.$ and $\left.\mathrm{C}^{18} \mathrm{O}\right)$ molecular emission relative to the dust emission in the inner disk region $(<50$ 
au radius). After re-calibration and inclusion of a previously omitted dataset, we have been able to significantly improve the signal-to-noise ratio (see Methods), revealing substructure that was only hinted at in the previous study.

Figure 1 shows the $1.33 \mathrm{~mm}$ thermal emission from the dust within a 100 au radius of the protostar. The dust emission peaks strongly within 5 au of the protostar and there is a shoulder ${ }^{9}$ at radius $\sim 20$ au, beyond which the dust optical depth suddenly decreases (Supplementary Figure 1). The dust emission is otherwise smooth without any indication of other dips, bumps or nonaxisymmetric features (such as observed towards, e.g., HL Tau ${ }^{11,12}$, TW Hya ${ }^{13}$ or Elias 2-27 ${ }^{14}$ ). From the thermal dust emission, we derive a lower limit to the total disk mass (dust + gas) of $0.01-0.04 M_{\text {Sun }}\left(10-40 M_{\text {Jup }} ;\right.$ see Methods).

The molecular gas emission is, meanwhile, much more extended than the dust continuum (Fig. 1); ${ }^{13} \mathrm{CO}$ and $\mathrm{C}^{18} \mathrm{O}$ emission is visible up to a radius of 70 au whereas the mm-dust emission is concentrated inside $40 \mathrm{au}^{9}$. The molecular gas emission is, however, only observed (with a spectral resolution of $0.35 \mathrm{~km} \mathrm{~s}^{-1}$ ) down to a radius of $\sim 15$ au at velocities $>2 \mathrm{~km} \mathrm{~s}^{-1}$ relative to the systemic velocity ${ }^{5}$ of $6.4 \mathrm{~km} / \mathrm{s}$ (Fig. 1). Velocity profile analysis using Keplerian masking of the ${ }^{13} \mathrm{CO}$ and $\mathrm{C}^{18} \mathrm{O}$ emission (Fig. 2) indicate a $0.4-0.8 M_{\text {Sun }}$ central protostar ${ }^{5,6,9}$.

Both ${ }^{13} \mathrm{CO}$ and $\mathrm{C}^{18} \mathrm{O}$ molecular gas emission are strongly suppressed inside a 15 au radius. If molecular line emission was present in this region, it would be Doppler-shifted with respect to the systemic velocity and would, therefore, not be obscured by the foreground material. Moreover, any gas along the line of sight would have difficulties absorbing the emission due to the large 
velocity and temperature gradient along the line of sight. Thus, the suppression must instead be a result of the local disk conditions. From the integrated molecular line emission, a gas mass of 0.03-0.07 $M_{\text {Sun }}\left(30-70 M_{\text {Jup }}\right.$; see Methods) is derived. Since the molecular emission is missing in the inner region, this value is, in fact, a lower limit to the gas mass if opaque dust is the cause of the suppression.

When radiation from a column of material (i.e., gas or dust) is observed at a particular wavelength, only emission that originates from line-of-sight optical depths $\lesssim 1$ is visible to the observer. All other radiation at the same wavelength, regardless of source, becomes obscured by the preceding material. As we are observing both molecular lines and dust $(1.33 \mathrm{~mm})$ continuum along the line of sight, if the dust column becomes optically thick (i.e., $\tau_{1.33 \mathrm{~mm}} \gtrsim 1$ ), the saturated continuum emission will overwhelm any line emission at a similar wavelength, even if it lies closer to the observer. The total mass (gas + dust) values presented above have large uncertainties due to the assumed gas-to-dust ratio and average temperature. As shown by Bjerkeli et al. ${ }^{9}$, the ${ }^{12} \mathrm{CO}$ emission at high velocities are detected spatially separated from the continuum emission. In addition, observed fundamental ro-vibrational ${ }^{15}$ lines of $\mathrm{CO}$ point to a hot $\mathrm{CO}$ gas reservoir in the inner $0.2 \mathrm{au}$, implying that UV/X-ray radiation originating from stellar accretion cannot be responsible for the entirely missing ${ }^{13} \mathrm{CO}$ and $\mathrm{C}^{18} \mathrm{O}$ emission. By comparing our observations with radiative transfer models ${ }^{16,17}$, the suppression of molecular line emission in the presence of dust permits us to infer the physical properties of this young disk and thus understand the relationship between the observed emission and the distribution of gas and dust in TMC1A. 
Our fiducial model is a parametrised protoplanetary disk with a standard dust population and canonical gas-to-dust ratio (see Methods). From the derived disk mass, our fiducial model predicts readily observable ${ }^{13} \mathrm{CO}$ and $\mathrm{C}^{18} \mathrm{O}$ gas emission from the inner $\sim 15$ au (Fig. 3, panel b); this model cannot provide the high optical depth required to suppress the molecular emission. Models of higher mass disks meanwhile suggest that a total disk mass of $>0.2 M_{\text {Sun }}\left(\sim 200 M_{\text {Jup }}\right.$; as compared to a $0.4-0.8 M_{\text {Sun }}$ protostar) is needed to reach the required optical depths to suppress the molecular emission (Fig. 3). Massive disks, however, are gravitationally unstable when the disk-to-stellar mass ratio $^{18}>10 \%$. In this case, the TMC1A disk should then be unstable and display non-axisymmetric structures ${ }^{14}$ on time scales of decades (see Methods), but neither the dust nor gas emission show evidence of such features. If non-axisymmetric structures are present in this disk, they are at lower amplitudes than probed by these observations.

A more plausible explanation for the large optical depth is that the dust population is not standard and large grains $(>1 \mathrm{~mm})$ are already present, at the very least, inside 30 au. Typically, dust mass absorption coefficients due to grains with icy mantles and sizes $\leq 0.25$ microns ${ }^{19}$ are sufficient to explain sub-millimetre thermal dust emission from protostellar envelopes and dense cores ${ }^{20,21}$, whereas larger grains are commonly used to reproduce dust emission from more evolved, Class II disks ${ }^{22,23}$. For TMC1A, larger dust grains in the inner regions increase the mass absorption coefficient ${ }^{24}$ and our modelling demonstrates that this can account for the suppression of molecular emission (Fig. 3) while also providing a lower disk-to-stellar mass ratio ( 5\%; see Methods). The suppression in molecular emission can also be reproduced if we adopt an unusually flat (i.e. a reduced vertical scale height; see Methods) and cold disk model. The properties of cold 
and flat disks however provide a better match to less massive disks around mature Class II sources, and are inconsistent with the thermal structure of such young disks ${ }^{25}$, especially that of TMC1A, which is known to still be accreting from the surrounding envelope ${ }^{6}$.

Following all of these considerations, we conclude that the presence of grains $\geq$ mm-size are the cause of the suppression of the molecular gas emission. Our spatially resolved data demonstrates the effect of large grains on the emergence of molecular gas emission on Solar System scales $(<30 \mathrm{au})$ in this young disk. In contrast to older Class II disks ${ }^{3}$, we find that the young protoplanetary disk around TMC1A has sufficient mass to form multiple Jupiter mass planets. The similarities in structure (e.g. scale height of the gas disk, radial exponential tail, surface density power-law index) and dust composition (small and large grains populations) inferred for this particular young, embedded protostellar disk, relative to more mature Class II disks, meanwhile implies that the seeds for planet formation are already present in the early stages of protoplanetary disk evolution.

Millimetre-size grains in such a young disk is of particular interest for planet formation. Core accretion models of giant planet formation rely on the availability of a large number of planetesimals $^{26}$ to explain the growth of planets. Astrophysical pebbles are, meanwhile, typically between $\mathrm{mm}$ - to $\mathrm{cm}-\mathrm{size}^{27}$. Pebbles accrete onto planetary bodies more efficiently than planetesimals owing to their much larger numbers and partial coupling to the gas, resulting in planetary growth time scales well within the disk lifetime ${ }^{28,29}$. Indeed, the presence of a significant population of mm-size dust in a $\sim 10^{5}$ year old disk is in agreement with time scales from recent models 
of dust growth ${ }^{1}$. It is also consistent with the measured size distribution of the thermally processed dust grains found in chondritic meteorites known as chondrules ${ }^{30}$ and, indeed, recent age estimates for the oldest known examples ${ }^{31}$. Suppression of molecular emission by dust provides an independent means to investigate the presence of grain growth in spatially resolved studies of young disks. Therefore, observations towards other protostellar systems similar to the one we presented here should be undertaken in order to determine how common mm-size dust is in the inner regions of very young disks, thus leading to a better understanding of when, and how frequently, young disks are forming planets. 


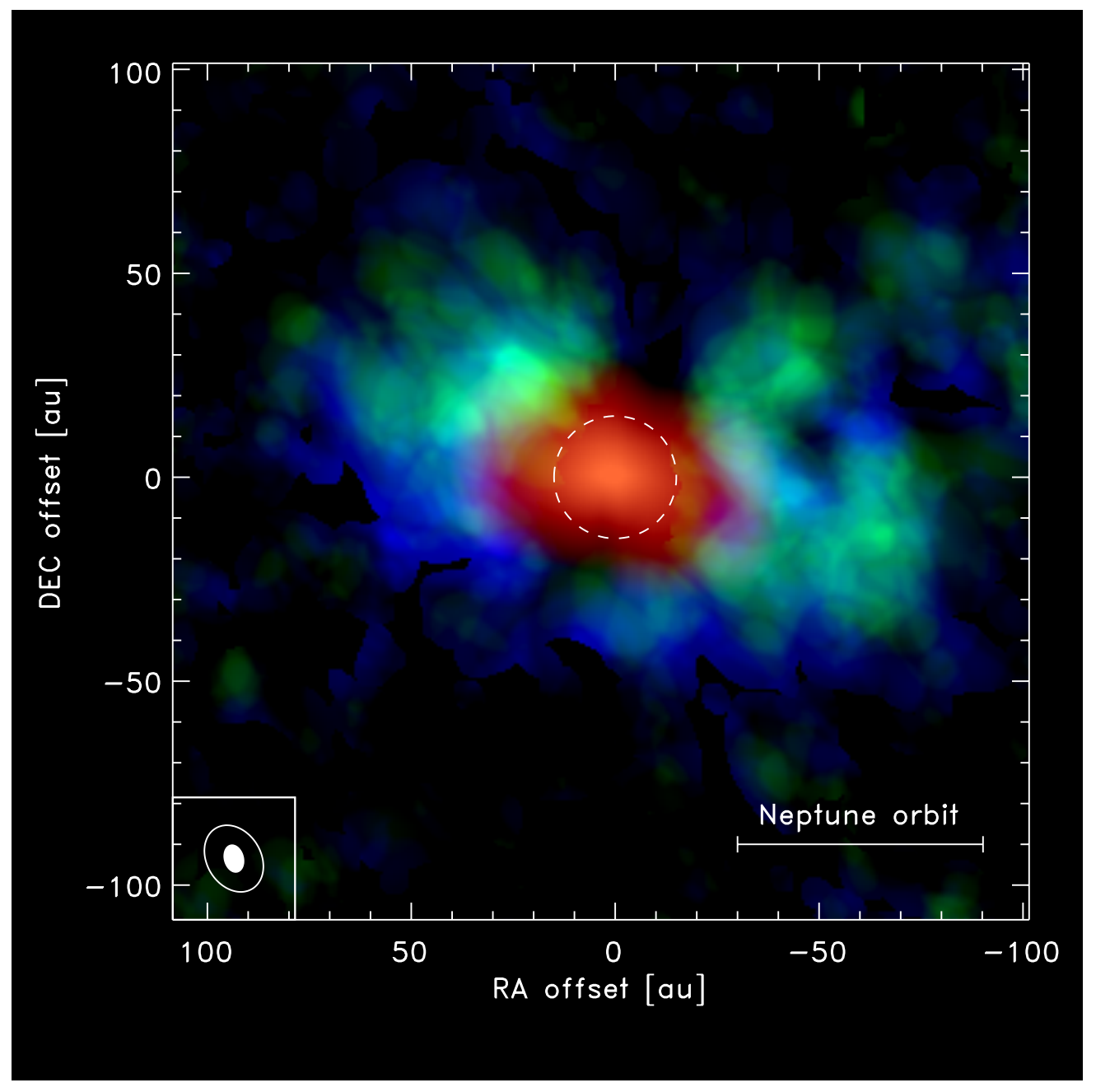

Figure 1 Dust continuum and integrated ${ }^{13} \mathrm{CO}$ and $\mathrm{C}^{18} \mathrm{O}$ emission map of TMC1A. The colours represent $1.3 \mathrm{~mm}$ dust continuum (red), ${ }^{13} \mathrm{CO}$ (green) and $\mathrm{C}^{18} \mathrm{O}$ (blue) zeroth moment maps. The intensities are scaled linearly with the dust continuum/integrated line 
fluxes. The peaks of the maps are $13.9 \mathrm{mJy}^{\text {beam }}{ }^{-1}, 33.8 \mathrm{mJy}$ beam $^{-1} \mathrm{~km} \mathrm{~s}^{-1}$, and $17.8 \mathrm{mJy}$ beam ${ }^{-1} \mathrm{~km} \mathrm{~s}^{-1}$ for the dust continuum, ${ }^{13} \mathrm{CO}$, and $\mathrm{C}^{18} \mathrm{O}$ zeroth moment maps, respectively. The molecular emission falls off steeply at radii $\lesssim 30$ au, and is completely absent in the inner 15 au as indicated by the dashed line contour. The diameter of Neptune's orbit $(60 \mathrm{au})$ is indicated at the bottom right. The synthesized beam sizes, shown in the bottom left, are $0.06^{\prime \prime} \times 0.05^{\prime \prime}$ (position angle of $25^{\circ}$ ) for the dust continuum and $0.12^{\prime \prime} \times 0.095^{\prime \prime}$ (position angle of $31^{\circ}$ ) for the molecular lines.

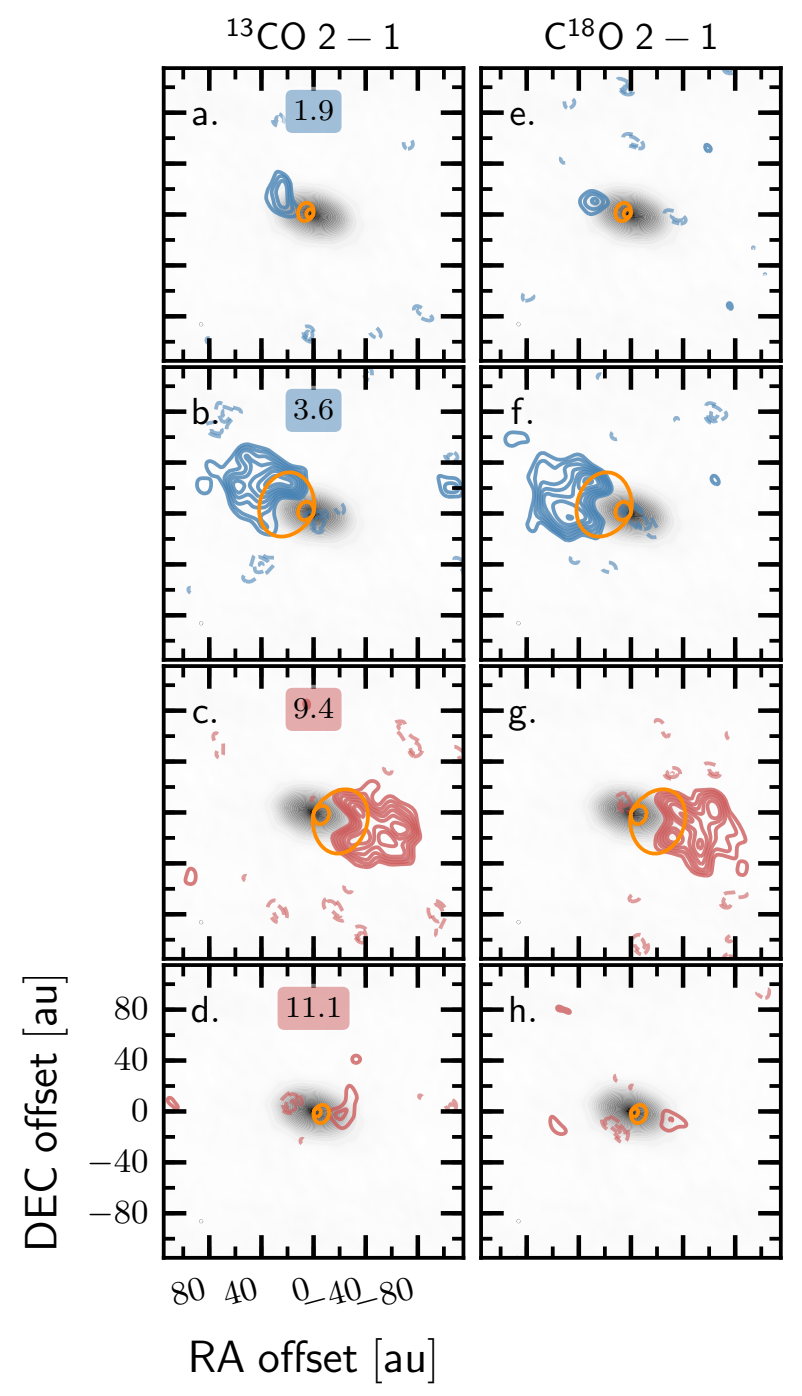

Figure $2{ }^{13} \mathrm{CO}$ and $\mathrm{C}^{18} \mathrm{O}$ channel maps. Line contours show integrated emission from 
$3 \sigma$ to $15 \sigma$ at every $\sigma$ for the ${ }^{13} \mathrm{CO}\left(\sigma=2.2 \mathrm{mJy}_{\text {beam }}{ }^{-1} \mathrm{~km} \mathrm{~s}^{-1}\right.$; panels $\left.\mathrm{a}-\mathrm{d}\right)$ and $\mathrm{C}^{18} \mathrm{O}$ channel maps ( $\sigma=1.6$ mJy beam ${ }^{-1} \mathrm{~km} \mathrm{~s}^{-1}$; panels $\mathrm{e}-\mathrm{h}$ ). Integrated maps begin at $1.0 \mathrm{~km} \mathrm{~s}^{-1}$ to $4.5 \mathrm{~km} \mathrm{~s}^{-1}$ for the blue side and $8.5 \mathrm{~km} \mathrm{~s}^{-1}$ to $12 \mathrm{~km} \mathrm{~s}^{-1}$ for the red side. Each panel shows the emission integrated over 5 channels (one channel is $0.35 \mathrm{~km} \mathrm{~s}^{-1}$ ). Absorption is denoted with dashed contours starting from $-5 \sigma$ up to $-2 \sigma$ in steps of $0.5 \sigma$. Dust continuum is shown in gray from $5 \sigma$ up to the maximum peak intensity. The midpoint velocity of each channel is shown at the top of each panel in the left column. Keplerian iso-velocity contours for a disk radius of 200 au and a $0.5 M_{\text {Sun }}$ central source inclined at $51^{\circ}$ with respect to the plane of the sky are shown in orange.
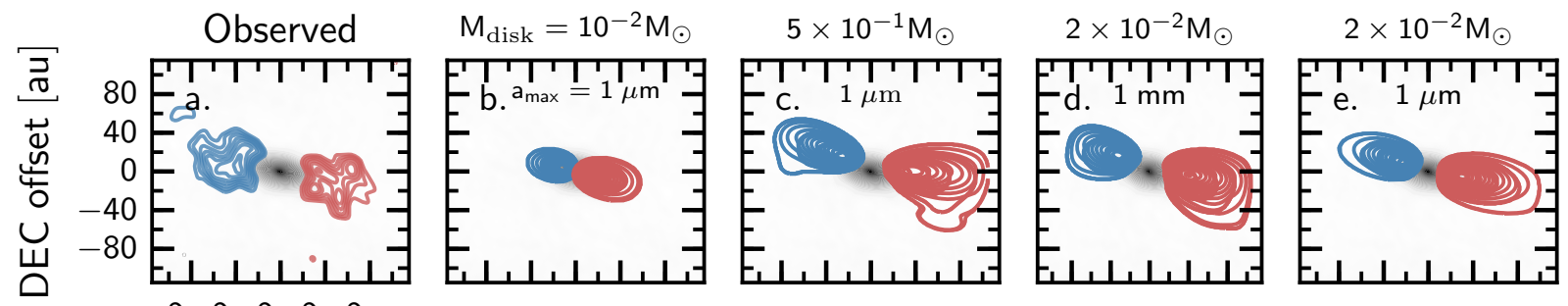
$8040 \quad 0_{4} 0_{8} 80$

RA offset [au]

Figure 3 Observed and simulated $\mathbf{C}^{18} \mathrm{O}$ emission maps. The molecular emission is integrated between 2 to $4.5 \mathrm{~km} \mathrm{~s}^{-1}$ (blue) and 8 to $10.5 \mathrm{~km} \mathrm{~s}^{-1}$ (red). The observed dust continuum is shown in gray with a linear scaling from $5 \sigma$ to the maximum. The observations are shown in panel (a). Four radiative transfer models $(b-e)$ with different parameters are shown: b) A disk with mass $0.01 M_{\text {Sun }}$; note that both red-and blue-shifted emission is expected to extend down to the central protostar. The depression in the molecular emission can be reproduced by c) a massive disk of $0.5 M_{\text {Sun }}$, d) a population 
of large grains $\left(a_{\max }=1 \mathrm{~mm}\right)$ or e) a flat, cold disk with parameters similar to more mature Class II sources. The adopted disk masses and maximum grain sizes are indicated in each panel. With the exception of panel (d), the models utilise small, bare dust grains $\left(a_{\max }=1 \mu \mathrm{m}\right)$ (see Methods). 


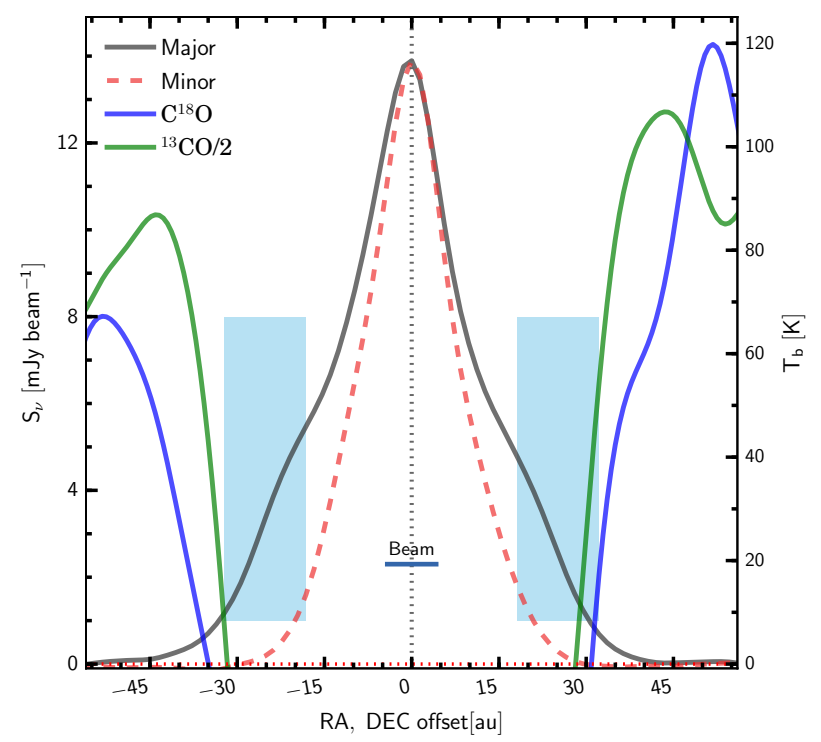

Supplementary Figure 1. Intensity profile of the dust continuum. The brightness profiles along major $\left(\mathrm{PA}=76^{\circ} \mathrm{E}\right.$ of $\mathrm{N}$; black) and minor $\left(\mathrm{PA}=166^{\circ} \mathrm{E}\right.$ of $\mathrm{N}$; red) axes of the disk are shown; the shoulder is located at $\sim 20$ au. The size of the beam is indicated at the bottom of the figure. The corresponding brightness temperature of the continuum is shown on the right axis. The blue and green lines show brightness profiles for the zeroth moment maps of $\mathrm{C}^{18} \mathrm{O}$ and ${ }^{13} \mathrm{CO}$ scaled by 2 , respectively, along the major axis. Two blue-shaded regions are shown to illustrate the approximate locations of the shoulder and the start of the gas emission hole.
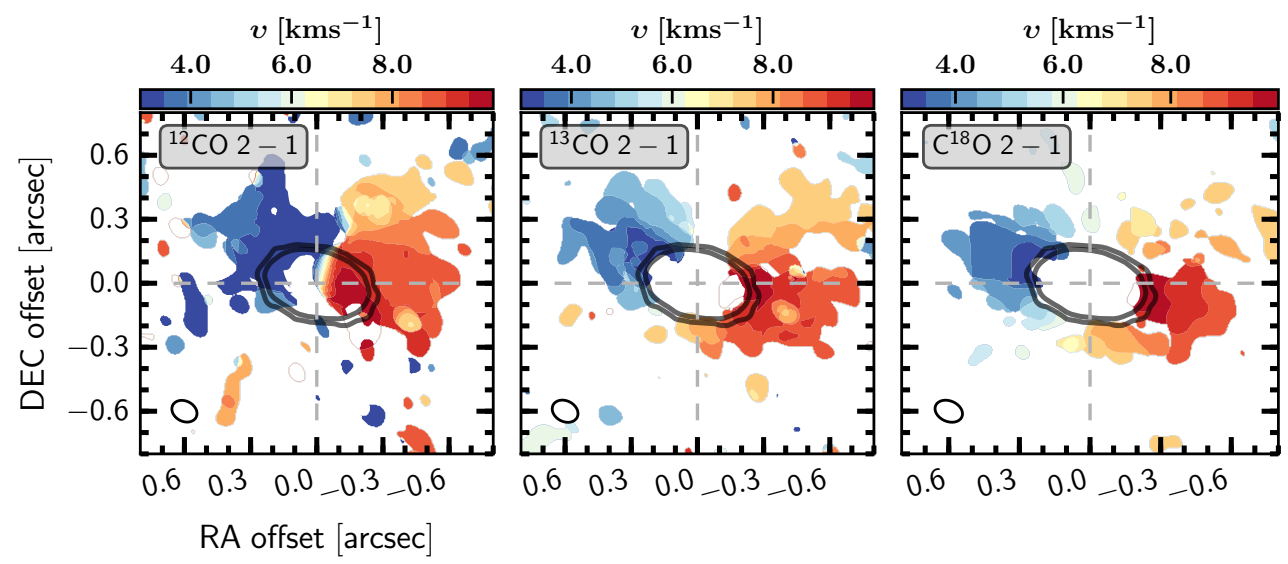

Supplementary Figure 2. First moment maps of the $\mathrm{CO}$ isotopologues. The blue- 
and red-shifted emission of the $\mathrm{C}^{18} \mathrm{O}$ (middle) and ${ }^{13} \mathrm{CO}$ (right) molecular emission show a clear rotating structure outside of the dust continuum, denoted with black contours. These maps were constructed only taking into account molecular emission from 2 to $11 \mathrm{~km} \mathrm{~s}^{-1}$. The ${ }^{12} \mathrm{CO}$ (left) first moment map shows rotating gas shifted north with respect to the $\mathrm{C}^{18} \mathrm{O}$ map. It also contains a highly blue-shifted gas component that is not seen in ${ }^{13} \mathrm{CO}$ or $\mathrm{C}^{18} \mathrm{O}$.

Acknowledgements This paper makes use of the following ALMA data: ADS/JAO.ALMA\#2015.1.01415.S. ALMA is a partnership of ESO (representing its member states), NSF (USA) and NINS (Japan), together with NRC (Canada) and NSC and ASIAA (Taiwan) and KASI (Republic of Korea), in cooperation with the Republic of Chile. The Joint ALMA Observatory is operated by ESO, AUI/NRAO and NAOJ. The authors would like to thank Allegro, the European ALMA Regional Centre node in the Netherlands, for providing the facilities and assistance in re-calibrating and imaging of the data. We thank the anonymous referees for their helpful comments that have greatly improved the manuscript. Furthermore, DH thanks Michiel Hogerheijde, Arthur Bosman and Ewine van Dishoeck for interesting discussions. DH is supported by EU ERC Advanced Grant 291141 "CHEMPLAN" and by a KNAW professor prize awarded to E. van Dishoeck. DH and LTM are part of Allegro, which is funded by the Netherlands Organisation for Scientific Research (NWO). PB acknowledge the support by the Swedish Research Council (VR) through contracts 2013-00472 and 2017-04924. The group of JKJ acknowledges support from the European Research Council (ERC) under the European Union's Horizon 2020 research and innovation programme (grant agreement No 646908) through ERC Consolidator Grant "S4F". Research at the Centre for Star and Planet Formation is funded by the Danish National Research Foundation (DNRF97). 
Author Contributions DH and LTM were responsible for the data re-calibration and reduction. DH was responsible for the analysis and wrote the manuscript together with PB, MHDvdW and JPR. MHDvdW and PB composed the observing proposal, with contributions from DH, JPR and JKJ. All authors contributed at various stages to the data analysis, discussed the results and contributed to the manuscript.

Author Information Reprints and permissions information is available at www.nature.com/reprints. The authors declare that they have no competing financial interests. Correspondence and requests for materials should be addressed to D. Harsono (email: harsono@strw.leidenuniv.nl).

Competing Interests The authors declare no competing interests.

\section{References}

1. Testi, L. et al. Dust Evolution in Protoplanetary Disks. In Beuther, H., Dullemond, C. \& Henning, T. (eds.) Protostars and Planets VI, 339-362 (Univ. of Arizona Press, Tucson, 2014).

2. Beckwith, S. V. W., Sargent, A. I., Chini, R. S. \& Guesten, R. A survey for circumstellar disks around young stellar objects. AJ 99, 924-945 (1990).

3. Pascucci, I. et al. A Steeper than Linear Disk Mass-Stellar Mass Scaling Relation. ApJ 831, 125 (2016).

4. The Extrasolar Planets Encyclopaedia. URL http://www.exoplanet.eu. Accessed 20-December-2017. 
5. Harsono, D. et al. Rotationally-supported disks around Class I sources in Taurus: disk formation constraints. A\&A 562, A77 (2014).

6. Aso, Y. et al. ALMA Observations of the Transition from Infall Motion to Keplerian Rotation around the Late-phase Protostar TMC-1A. ApJ 812, 27 (2015).

7. Lada, C. J. Star formation - From OB associations to protostars. In Peimbert, M. \& Jugaku, J. (eds.) Star Forming Regions, vol. 115 of IAU Symposium, 1-17 (1987).

8. Hogerheijde, M. R., van Dishoeck, E. F., Blake, G. A. \& van Langevelde, H. J. Envelope Structure on 700 AU Scales and the Molecular Outflows of Low-Mass Young Stellar Objects. ApJ 502, 315-336 (1998).

9. Bjerkeli, P., van der Wiel, M. H. D., Harsono, D., Ramsey, J. P. \& Jørgensen, J. K. Resolved images of a protostellar outflow driven by an extended disk wind. Nature 540, 406-409 (2016).

10. Chandler, C. J., Terebey, S., Barsony, M. \& Moore, T. J. T. The Small-Scale Outflow Structure of Embedded Sources in Taurus. Astrophysics \& Space Science 224, 109-112 (1995).

11. ALMA Partnership et al. The 2014 ALMA Long Baseline Campaign: First Results from High Angular Resolution Observations toward the HL Tau Region. ApJL 808, L3 (2015).

12. Zhang, K., Blake, G. A. \& Bergin, E. A. Evidence of Fast Pebble Growth Near Condensation Fronts in the HL Tau Protoplanetary Disk. ApJL 806, L7 (2015).

13. Andrews, S. M. et al. Ringed Substructure and a Gap at 1 au in the Nearest Protoplanetary Disk. ApJL 820, L40 (2016). 
14. Pérez, L. M. et al. Spiral density waves in a young protoplanetary disk. Science 353, 15191521 (2016).

15. Herczeg, G. J., Brown, J. M., van Dishoeck, E. F. \& Pontoppidan, K. M. Disks and outflows in $\mathrm{CO}$ rovibrational emission from embedded, low-mass young stellar objects. A\&A 533, A112 (2011).

16. Bruderer, S., van Dishoeck, E. F., Doty, S. D. \& Herczeg, G. J. The warm gas atmosphere of the HD 100546 disk seen by Herschel. Evidence of a gas-rich, carbon-poor atmosphere? A\&A 541, A91 (2012).

17. Robitaille, T. P. HYPERION: an open-source parallelized three-dimensional dust continuum radiative transfer code. $A \& A \mathbf{5 3 6}, \mathrm{A} 79$ (2011).

18. Lodato, G. \& Rice, W. K. M. Testing the locality of transport in self-gravitating accretion discs. MNRAS 351, 630-642 (2004).

19. Ossenkopf, V. \& Henning, T. Dust opacities for protostellar cores. A\&A 291, 943-959 (1994).

20. Henning, T., Michel, B. \& Stognienko, R. Dust opacities in dense regions. P\&SS 43, 13331343 (1995).

21. Launhardt, R. et al. The Earliest Phases of Star Formation (EPoS): a Herschel key project. The thermal structure of low-mass molecular cloud cores. A\&A 551, A98 (2013).

22. Ricci, L. et al. Dust properties of protoplanetary disks in the Taurus-Auriga star forming region from millimeter wavelengths. $A \& A \mathbf{5 1 2}$, A15 (2010). 
23. Andrews, S. M. Millimeter-Radio Observations of the Hallmarks of Planet Formation in Circumstellar Disks. In Kawabe, R., Kuno, N. \& Yamamoto, S. (eds.) New Trends in Radio Astronomy in the ALMA Era: The 30th Anniversary of Nobeyama Radio Observatory, vol. 476 of Astronomical Society of the Pacific Conference Series, 149-156 (2013).

24. Andrews, S. M., Wilner, D. J., Hughes, A. M., Qi, C. \& Dullemond, C. P. Protoplanetary Disk Structures in Ophiuchus. ApJ 700, 1502-1523 (2009).

25. Visser, R., van Dishoeck, E. F., Doty, S. D. \& Dullemond, C. P. The chemical history of molecules in circumstellar disks. I. Ices. A\&A 495, 881-897 (2009).

26. Mizuno, H. Formation of the Giant Planets. Progress of Theoretical Physics 64, 544-557 (1980).

27. Lambrechts, M. \& Johansen, A. Forming the cores of giant planets from the radial pebble flux in protoplanetary discs. A\&A 572, A107 (2014).

28. Lambrechts, M. \& Johansen, A. Rapid growth of gas-giant cores by pebble accretion. $A \& A$ 544, A32 (2012).

29. Ida, S., Guillot, T. \& Morbidelli, A. The radial dependence of pebble accretion rates: A source of diversity in planetary systems. I. Analytical formulation. A\&A 591, A72 (2016).

30. Davis, A. M. et al. Samples of the Solar System: Recent Developments. In Beuther, H., Dullemond, C. \& Henning, T. (eds.) Protostars and Planets VI, 809-831 (Univ. of Arizona Press, Tucson, 2014). 
31. Connelly, J. N. et al. The Absolute Chronology and Thermal Processing of Solids in the Solar Protoplanetary Disk. Science 338, 651-655 (2012).

\section{Methods}

Observations and calibration. TMC1A was observed with ALMA on three different dates: October 162015 (Execution Block 1; EB1), October 232015 (EB2) and October 302015 (EB3). The last two execution blocks were taken under excellent weather conditions (water vapour $<1$ mm). Bjerkeli et al. ${ }^{9}$ presented only execution blocks EB2 and EB3 with lower water vapour and relatively good stability. Three SPWs were dedicated to observe the ${ }^{12} \mathrm{CO} 2-1\left(230.538 \mathrm{GHz}, E_{\text {up }}\right.$ $=16.60 \mathrm{~K}),{ }^{13} \mathrm{CO} 2-1\left(220.399 \mathrm{GHz}, E_{\mathrm{up}}=15.87 \mathrm{~K}\right)$, and $\mathrm{C}^{18} \mathrm{O} 2-1\left(219.560 \mathrm{GHz}, E_{\text {up }}=15.81\right.$ K) transitions. As poor conditions can lead to defects in images ${ }^{32}$, the phase centre of TMC1A was derived from the EB3 data, which had the best atmospheric stability.

The entire data set (all three EBs) were re-calibrated with CASA v4.7.2. Instead of using the observed integration time $(3 \mathrm{~s})$ to obtain the phase solutions, we opted to use $12 \mathrm{~s}$ in order to obtain better solutions with higher signal-to-noise. The flux calibrators were manually checked for consistency between execution blocks. Furthermore, we have used a non-standard water-vapour radiometer (WVR) scaling ${ }^{33}$ to improve the phase delays for each antenna due to atmospheric water content. Scaling of the WVR solutions is the only way to improve the phase stability on very short timescales (integration time) which is typically not possible except for very strong targets ${ }^{33}$. We refer to Bjerkeli et al. ${ }^{9}$ for imaging details. 
Since the $1.3 \mathrm{~mm}$ continuum emission of TMC1A is detected with a high image signal-tonoise $(S / N>100)$, it is possible to calibrate the data set against itself ("self-calibration"). The improvements introduced by WVR corrections resulted in higher signal-to-noise data and images, allowing us to perform self-calibration on the target down to a 20 s timescale. At first, large time scale phase corrections ( 5 minutes) were applied to all three data sets in order to minimize the long period atmospheric phase decoherence. After the final self-calibration, the phase de-coherence that usually spreads flux around an image with increasing noise ${ }^{32}$ was reduced. The resulting noise levels amount to an improvement of a factor four and two relative to the previously presented continuum image and $\mathrm{CO}$ maps $^{9}$, respectively.

Thermal dust emission. The dust continuum emission provides a view into the density structure of the disk. After calibration and analysis, the resulting synthesized beam $\left(0.06^{\prime \prime} \times 0.05^{\prime \prime}\right.$, $\left.\mathrm{PA}=25^{\circ}\right)$ is sufficient to spatially resolve the full extent of the dust disk $\left(\sim 0.3^{\prime \prime}\right.$ radius $)$. Due to the small, synthesized beam, a common fixed phase centre was introduced to minimize imaging artefacts due to phase centre offsets between execution blocks. The peak of the continuum intensity is $13.9 \mathrm{mJy}_{\text {beam }}^{-1}$ (Figure 1) with an integrated flux density of $145 \pm 2 \mathrm{mJy}$ at $225 \mathrm{GHz}$ inside a $1^{\prime \prime}$ box. The image noise level is $45 \mu \mathrm{Jy}$ beam $^{-1}$. The disk is inclined at $51^{\circ}$ with respect to the plane of the sky $\left(0^{\circ} \text { is face-on }\right)^{5,6,9}$, as derived from the ratio of the major to minor axis (deconvolved $\left.F W H M=0.24^{\prime \prime} \times 0.15^{\prime \prime}\right)$ of the dust continuum image. The PA was determined from a $2 \mathrm{D}$ Gaussian fit to the emission. Supplementary Figure 1 shows the brightness profile along the major $\left(\mathrm{PA}=76^{\circ}\right)$ and minor $\left(\mathrm{PA}=166^{\circ}\right)$ axes; it is symmetric up to $\sim 20 \mathrm{au}$, where a shoulder is visible 
due to a change in the optical depth.

The dust continuum is observed at $225 \mathrm{GHz}(1.33 \mathrm{~mm})$, and may be contaminated by freefree emission from unresolved accretion shocks at the stellar surface. However, Scaife et al. ${ }^{34}$ have shown that the mm-continuum emission from Class I sources similar to TMC1A are instead dominated by the dust thermal emission ${ }^{35}$. By comparing the images obtained from applying different weightings (superuniform versus Briggs weighting ${ }^{36}$ of 1), at most, a flux of 3 mJy can be attributed to free-free emission, which is negligible compared to the total flux of the disk. Therefore, the total disk mass (gas + dust) can be safely estimated from the dust continuum by assuming an average dust temperature and total mass absorption coefficient $\kappa_{\nu}$. Under the assumption of optically thin dust thermal emission, the total mass is given by:

$$
M_{\text {total=gas }+ \text { dust }}=\frac{S_{\nu} d^{2}}{\kappa_{\nu} B_{\nu}\left(T_{\mathrm{d}}\right)},
$$

where $S_{\nu}$ is the flux density in Janskys, $\kappa_{\nu}$ is the mass absorption coefficient at frequency $\nu$ corrected for a standard gas-to-dust mass ratio $^{37}(100)$ and $B_{\nu}$ is the Planck function at the dust temperature $T_{\mathrm{d}}$ assuming the gas temperature is coupled to the dust.

One of the quantities typically used to estimate the evolutionary stage of a protostellar system is the disk mass ${ }^{38-40}$. A total disk mass (gas+dust) of $0.012-0.036 M_{\text {Sun }}$ is derived using dust temperatures in the 30 to $80 \mathrm{~K}$ range as indicated by the measured brightness temperature $\left(T_{\mathrm{B}}=\frac{\lambda^{2}}{2 k_{\mathrm{B}}} I_{\nu}=30-120 \mathrm{~K}\right.$ in the inner $\left.30 \mathrm{au}\right)$. The adopted total mass absorption coefficient ${ }^{2}$ is $\kappa_{\nu}=0.01(\nu / 230 \mathrm{GHz}) \mathrm{cm}^{2} \mathrm{~g}^{-1}$, which is similar to a $\kappa_{\nu}$ value of $0.0085 \mathrm{~cm}^{2} \mathrm{~g}^{-1}$ at $1.3 \mathrm{~mm}$ from Ossenkopf \& Henning ${ }^{19}$. For mature Class II disks, a mass absorption coefficient of $\sim 0.02 \mathrm{~cm}^{2}$ 
$\mathrm{g}^{-1}$ is typically used ${ }^{22,41}$. By adopting dust opacities appropriate for Class II disks, a factor of two lower disk mass is obtained for TMC1A.

Recent studies of the unresolved dust continuum and multi-wavelength spectral index have found evidence for grain growth in Class 0 and I objects ${ }^{34,42-47}$. Indeed, Lee et al. ${ }^{48}$ recently found evidence for an optically thick dust continuum in the edge-on protostellar system HH 212. However, the observation of emission from the surface layers of a disk in such a configuration is not trivial. In part due to its lower inclination, the surface layers of the TMC1A disk are directly observable and permit the observation of the optically thick dust continuum and the subsequent effect on the emergence of molecular emission.

Dust mass absorption coefficients and properties. We explored the effects of various dust mass absorption coefficients (opacities) on the emergence of molecular emission. They were calculated using the Bohren and Huffman Mie ("BHMie") $\operatorname{code}^{49}$ distributed with Hyperion ${ }^{17}$. For small grains, the sizes are between $0.01-1$ microns following Andrews et al. ${ }^{41}$. For large grain opacities, we vary the maximum grain size, $a_{\max }$, between $1 \mathrm{~mm}$ and $10 \mathrm{~cm}$. Dust opacities with $a_{\max }=10$ $\mathrm{cm}$ lead to a lower dust mass absorption coefficient at millimetre wavelengths. The opacities presented here are normalised to the dust mass. The limited wavelength coverage of our data set can only constrain the maximum grain size to a few millimeters. Thus, we adopt $a_{\max }$ of $1 \mathrm{~mm}$ for simplicity. Optical constants were taken from Weingartner \& Draine ${ }^{50}$ and Draine ${ }^{51}$.

We have compared our calculated opacities with Ossenkopf \& Henning opacities ${ }^{19}$ with a standard $^{52}$ size distribution and a thin ice mantle at densities of $10^{6} \mathrm{~cm}^{-3}$. The mass absorption 
coefficient differs by a factor of two $\left(0.899 \mathrm{~cm}^{2} \mathrm{~g}^{-1}\right.$ with ice mantle versus $\sim 2 \mathrm{~cm}^{2} \mathrm{~g}^{-1}$ without $)$ at $1.33 \mathrm{~mm}$. While Ossenkopf \& Henning opacities describe the dust properties of protostellar objects, BHMie calculations are suitable for T-Tauri disks ${ }^{2,53}$. Since the presence of ice lowers the mass absorption coefficient, in order to maximize the absorption coefficient, only bare grains are considered. The use of bare grains furthermore permit the derived disk mass to be minimized. Despite these considerations, Ossenkopf \& Henning opacities alone cannot provide the larger maximum grain size required to reach the derived optical depths.

Molecular emission. Figure 1 (blue and green colours) shows the zeroth moment maps for the observed $\mathrm{CO}$ isotopologue lines. Moment maps were constructed by considering pixels whose intensities $>3 \sigma\left(1 \sigma=2.1,2.3\right.$, and $1.7 \mathrm{mJy}_{\text {beam }}{ }^{-1}$ per $0.35 \mathrm{~km} \mathrm{~s}^{-1}$ channel for ${ }^{12} \mathrm{CO},{ }^{13} \mathrm{CO}$, and $\mathrm{C}^{18} \mathrm{O}$, respectively) and velocity channels between $2-11 \mathrm{~km} \mathrm{~s}^{-1}$. For comparison with the known wind component ${ }^{9}$, which is best traced by the ${ }^{12} \mathrm{CO} 2-1$ line, the line profiles of the three $\mathrm{CO}$ isotopologs were extracted from a $2^{\prime \prime}$ box (from $-1^{\prime \prime}$ to $1^{\prime \prime}$ ). ${ }^{12} \mathrm{CO}$ molecular emission is detected between $-3.8-14.4 \mathrm{~km} \mathrm{~s}^{-1}$. On the other hand, the $\mathrm{C}^{18} \mathrm{O} 2-1$ line is detected between $2.15-10.55$ $\mathrm{km} \mathrm{s}^{-1}$ and traces the quiescent gas of the Keplerian disk. The characteristic butterfly pattern of a Keplerian velocity profile is clearly visible in the channel maps (Figure 2 and Supplementary Figure 2). Both zeroth (Figure 1) and first (Supplementary Figure 2) moment maps show that ${ }^{13} \mathrm{CO}$ and $\mathrm{C}^{18} \mathrm{O}$ emission is absent within the inner $15 \mathrm{au}$.

The integrated line fluxes for ${ }^{13} \mathrm{CO}$ and $\mathrm{C}^{18} \mathrm{O}$ lines are $0.99 \pm 0.2 \mathrm{Jy} \mathrm{km} \mathrm{s}{ }^{-1}$ and $1.04 \pm 0.1 \mathrm{Jy}$ $\mathrm{km} \mathrm{s}^{-1}$, respectively, within a $1^{\prime \prime}$ box. The $1 \sigma$ error was determined from the sum of an averaged 
root mean square (r.m.s.) over the spectral range including a $20 \%$ flux error. In comparison to the observations of Harsono et al. ${ }^{5}$, the similarity in the ${ }^{13} \mathrm{CO}$ and $\mathrm{C}^{18} \mathrm{O}$ fluxes is due to the missing short spacing. It was already hinted at that the $\mathrm{C}^{18} \mathrm{O}$ emission is more compact than the ${ }^{13} \mathrm{CO}$ emission; thus, ${ }^{13} \mathrm{CO}$ is affected by the resolved-out large structure ${ }^{5}$ more strongly than the $\mathrm{C}^{18} \mathrm{O}$. Assuming optically thin emission, adopting a $\mathrm{CO}$ abundance of $10^{-4}$ with respect to $\mathrm{H}_{2}$, a ${ }^{16} \mathrm{O} /{ }^{18} \mathrm{O}$ isotopic ratio ${ }^{54}$ of 560 and a rotational temperature between 40 to $80 \mathrm{~K}^{55}$, the $\mathrm{C}^{18} \mathrm{O}$ line gives a derived gas mass of $0.03-0.07 M_{\text {Sun }}$. These values are roughly comparable to the gas mass derived from the dust continuum flux assuming a gas to dust ratio of 100 . We note that a lower CO abundance would result in an even higher disk mass.

To obtain the disk structure, we have fitted the continuum image using Least Square Minimization ${ }^{56}$ and a Markov Chain Monte Carlo package ("emcee"57). The minimization is performed on pixels whose intensities $\gtrsim 5 \sigma$. The intensity profile is fitted with a power-law disk including an exponential taper:

$$
\begin{aligned}
& \Sigma(R)=\Sigma_{0}\left(\frac{R}{R_{0}}\right)^{-p} \exp \left(-\left(\frac{R}{R_{0}}\right)^{2-p}\right) ; \\
& T(R)=T_{0} R^{-q} .
\end{aligned}
$$

We find that a disk characterized by $p \sim 0.6_{-0.1}^{+0.1}$ and $q \sim 0.6_{-0.1}^{+0.1}$ can reproduce the continuum intensity profile. We also tried a simple two-grain population model:

$$
\Sigma_{\text {tot }}=\Sigma_{\text {small }}+\Sigma_{\text {large }}
$$

where each $\Sigma$ is described by Equation 2 . This returned a flat $p \lesssim 0$ as the best-fitting model. For completeness, we also tried disk models without a radial taper. Such models favour a steep radial 
power-law index, $p \sim 5$, in order to obtain a steep mass distribution that achieves the necessary high optical depth at the shoulder ( $\sim 15 \mathrm{au})$. Guided by the above parameters, we also generated a flat, thin disk model following Murillo et al. ${ }^{58}$ and a density profile that follows Equation 2 . These models prefer $p \sim 0.7_{-0.3}^{+0.4}$ and $q \sim 0.9_{-0.2}^{+0.1}$ given an opacity described by a combined population of small and large grains. Visibility fitting ${ }^{5}$ using power-law disk structures with different values of $p$ provided disk parameters that are consistent with the above results.

Our image fitting does not fully explore all of the possible parameter space. Instead, it provides guidance for $2 \mathrm{D}$ self-consistent radiative transfer models that we used to understand the emergence of the molecular emission ${ }^{16}$. For simplicity, we chose a power-law index of $p=1$ scaled by the mass derived from the observations; a value of $p=0.5$, meanwhile, produces an intensity profile that is too shallow. The disk gas scale height, $H$, is set to 0.12 au at 1 au. Based on the dust, hydrostatic equilibrium for the TMC1A disk predicts a scale height of 0.03 au. However, we found that the gas and the small grains could be characterized with a different scale height as compared to a mm-sized dust grain distribution. Our goal with the radiative transfer modelling is not to provide a best-fit model to the data, but instead to demonstrate how the different parameters affect the emergence of $\mathrm{CO}$ emission. The Monte Carlo frequency-dependent radiative transfer code, Hyperion ${ }^{17}$, was used to derive the temperature structure for the various dust opacities described above. A stellar luminosity of $2.7 L_{\mathrm{Sun}}$ and an effective stellar temperature of $4000 \mathrm{~K}$ were adopted ${ }^{59}$. The gas temperature is assumed to be perfectly coupled to the dust temperature. Self-consistent gas temperature calculations lead to a warm molecular layer and will result in a stronger molecular emission, however, we do not observe this effect. There is evidence that points 
towards lower CO abundances in Class II disks ${ }^{16,60-63}$, but not necessarily toward younger, Class I disks (van’t Hoff et al. in press, de la Villarmois et al. in press). An escape probability molecular excitation code ${ }^{16}$ with adaptive gridding (to resolve the physical and velocity structure of the disk) was used to simulate the $\mathrm{CO}$ line emission. Collisional rate coefficients and Einstein $A$ values were obtained from the Leiden Molecular Database ${ }^{64,65}$, extrapolated ${ }^{66}$ to $J=60$ to account for the high gas temperatures in the inner few au. The partition function is self-consistently calculated by taking the populated $J$ levels into account.

Our fiducial disk model has a mass of $10^{-2} M_{\text {Sun }}$ and uses small grain dust opacities (Fig. 3b). This model predicts observable red- and blue-shifted molecular emission in the inner 15 au, in contrast with the observations. We intentionally avoid comparing the molecular emission close to the systemic velocity since the observations are highly dependent on the baseline coverage of the data set. Via additional models, we explored the effects of the disk mass, disk flaring angle and dust properties.

We find that the suppression of the molecular emission can be reproduced if the disk mass $>2 \times 10^{-1} M_{\text {Sun }}$ (Fig. 3c). More specifically, the optical depth of the $\mathrm{C}^{18} \mathrm{O}$ line can easily be as high as 10 , which results in a gas mass of $\sim 2 \times 10^{-1} M_{\text {Sun }}$. A low mass disk $\left(\sim 10^{-2} M_{\text {Sun }}\right)$ with large grain opacities, but otherwise standard parameters, also reproduces the suppression in molecular emission (Fig. 3d). As the mass estimates presented earlier were calculated under the assumption of optical thinness, this implies that these masses should be considered approximate.

A thick, flared disk model (scale height $H$ of 0.3 au at $1 \mathrm{au}$ ) predicts observable molecular 
emission inside 15 au radius. In a such a flared disk, the location of the gas emission is always above the dust photosphere (optical depth $\sim 1$; where the emission originates). In the optically thick region of the disk, the peak of the observed emission is related to the gas excitation temperature and the temperature at the dust photosphere:

$$
T_{\text {observed }}=T_{\text {excitation }}-T_{\text {dust }},
$$

where the intensity is given in terms of temperature since it is thermalised $\left(S=B_{\nu}(T)\right)$ and at the Rayleigh-Jeans limit. If the two temperatures are similar, the continuum-subtracted gas emission is directly related to the difference in kinetic temperature. The peak of the gas emission is thus given by $S_{\nu}=B_{\nu}\left(T_{\text {excitation }}-T_{\text {dust }}\right)$, which results in negligible molecular emission. The typical noise level per beam translates to $\gtrsim 4 \mathrm{~K}$ uncertainties in the temperature, which mean that our data is sensitive to a $4 \mathrm{~K}$ difference between the dust and gas emitting layers. A flat disk $(H / R=$ 0.06 Fig. 3e), meanwhile, produces an absence of emission inside 15 au since the gas and dust are vertically confined to a small region with a small temperature difference between the two emitting surfaces/photospheres. However, such a flat disk is typically found in lighter, mature Class II disks $^{24}$ and not in massive, young disks such as TMC1A.

A temperature inversion due to strong viscous heating in the disk could explain the absence of molecular emission. In accreting disk models ${ }^{67}$, the inversion is typically found in regions of the disk $>300 \mathrm{~K}$. To obtain a temperature inversion at a distance of 15 au in TMC1A, a very high stellar accretion rate $\left(>10^{-5} M_{\text {Sun }} \mathrm{yr}^{-1}\right.$ ) is required. This yields a total luminosity $>10 L_{\text {Sun }}$, which is inconsistent with the measured bolometric luminosity ${ }^{68}$ of $2.7 L_{\odot}$. Furthermore, modelling of the water lines ${ }^{69}$ observed by Herschel $H I F I^{68,70}$ also suggests accretion rates of $10^{-6} M_{\odot} \mathrm{yr}^{-1}$. 
Therefore, a temperature inversion is unlikely to be responsible for the missing molecular lines.

Dynamical mass of the young star and disk stability. The dynamical mass of a protostellar object places important constraints on the evolution and structure of the associated protoplanetary disk. The disk-to-stellar mass ratio also dictates the gravitational stability of the disk ${ }^{18,71}$. Figure 2 shows the Keplerian iso-velocity contours against the ${ }^{13} \mathrm{CO}$ and $\mathrm{C}^{18} \mathrm{O}$ emission within the same velocity range. Supplementary Figure 2 meanwhile shows the first moment map indicating the direction of the Keplerian rotation. The iso-velocity contours and subsequent Keplerian velocity profile $^{9}$ indicates that the protostellar mass is between $0.4-0.8 M_{\text {Sun }}$. The range in mass results from the consideration that the line emission is not optically thin and, since the highly Dopplershifted emission near the protostar is absent, we are limited from placing better constraints on the protostellar mass.

Using the disk masses derived from both gas and dust emission, the disk-to-stellar mass ratio ranges from 0.02 to 0.04 . As noted above, the disk mass must be higher $\left(>0.2 M_{\text {Sun }}\right)$ in order to suppress the molecular emission if a standard grain population is used. Such a high disk mass would imply that the disk-to-stellar mass ratio is between 0.25 to 0.625 . Numerical simulations ${ }^{72,73}$ show that such a high disk-to-stellar mass ratio is gravitationally unstable disk and leads to non-axisymmetric structures. The $\alpha$-viscosity ${ }^{74}$ of such a disk is of the order 0.05 to 0.1 , while Kratter et al. ${ }^{75}$ report $\alpha$ values between 0.3 to 0.8 for non-fragmenting disks. The lifetime of a disk under such high viscosity is on the order of $10^{4} \mathrm{yr}$ at 20 au $\left(t_{\mathrm{visc}}=R^{2} / \alpha c_{\mathrm{s}}\right)$. Furthermore, non-axisymmetric structures should develop on dynamical time scales $(\sim 9-20$ years $)$ at the radii being considered ${ }^{18}$. Since non-axisymmetric structures are not observed in either gas or dust, this 
implies the disk is stable and has a lower disk-to-stellar mass ratio.

Effectiveness of photo-destruction. Given a $0.4 M_{\text {Sun }}$ star with an effective temperature of 4000 $\mathrm{K}^{59}$, a bolometric luminosity $2.7 L_{\mathrm{Sun}}$ and a blackbody spectral energy distribution, our modelling predicts that it is not possible to substantially photodissociate $\mathrm{CO}$ in the disk out to 15 au, regardless of the vertical structure or disk's mass distribution. For these stellar properties, photodissociation of $\mathrm{CO}$ and its isotopologues is only efficient near the disk inner edge $(<0.5 \mathrm{au})^{76}$. Furthermore, high-velocity ${ }^{12} \mathrm{CO} 2-1$ molecular emission is observed inside a radius of 15 au but above the disk surface (in the outflow) ${ }^{9}$. This suggests that ${ }^{12} \mathrm{CO}$ molecules are present in the disk inside 15 au. Fundamental CO ro-vibrational lines have also been detected in both absorption and emission toward $\mathrm{TMC} 1 \mathrm{~A}^{15}$, and were shown to originate from within $0.2 \mathrm{au}$.

Following Visser et al. ${ }^{77}$, a blackbody of $4000 \mathrm{~K}$ with the addition of 1 Draine field removes $\mathrm{CO}$ up to $A_{\mathrm{v}}=2.3$. Since the photodissociation rate $^{78}$ is $k_{\text {diss }} \propto \exp \left(-\gamma A_{\mathrm{v}}\right)$, where $\gamma$ is an attenuation factor and $A_{\mathrm{V}}$ is the visual extinction, the amount of UV radiation necessary to photodissociate a molecule increases exponentially with column density. By calculating the vertical $A_{\mathrm{v}}$ at different radii, we determine that 1 Draine field can only dissociate $10^{-4}$ of the total CO column at 14 au. This only effectively destroys $\mathrm{CO}$ on the disk surface at $>5$ scale heights above the disk midplane. To entirely dissociate CO would require $\sim 10^{4}$ Draine fields. X-rays from accretion, meanwhile, tend to produce a warm ionized layer at the surface which increases the amount of $\mathrm{H}_{3}^{+}$, which readily destroys $\mathrm{CO}$ to form $\mathrm{HCO}^{+}$. Such chemistry drives the formation of $\mathrm{HCO}^{+}$on the warm surface layers while decreasing the $\mathrm{CO}$ abundance. Nevertheless, even if we adopt a low ${ }^{12} \mathrm{CO}$ 
relative abundance of $10^{-6}$, we still predict that $\mathrm{CO}$ should be observable inside 15 au. In either case, for reasonable UV and X-ray intensities, the reduction of $\mathrm{CO}$ abundance in the disk surface layers is simply not enough to explain the observed hole in the molecular emission.

Code Availability Hyperion is a public Monte Carlo continuum radiative transfer that can be downloaded from http://www.hyperion-rt.org/. It also contains a routine to calculate the dust opacities. The primary line radiative transfer code is described in Bruderer et al. ${ }^{16}$. The code is not public due to the lack of documentation and its non-trivial usage.

Data availability The datasets generated and/or analysed during the current study are available in the ALMA archive, https://almascience.nrao.edu/alma-data/archive, and from the corresponding author.

\section{Additional References}

32. Carilli, C. L. \& Holdaway, M. A. Tropospheric phase calibration in millimeter interferometry. Radio Science 34, 817-840 (1999).

33. Maud, L. T. et al. Phase correction for ALMA. Investigating water vapour radiometer scaling: The long-baseline science verification data case study. A\&A 605, A121 (2017).

34. AMI Consortium et al. Radio continuum observations of Class I protostellar discs in Taurus: constraining the greybody tail at centimetre wavelengths. MNRAS 420, 3334-3343 (2012).

35. Ubach, C. et al. Radio monitoring of protoplanetary discs. MNRAS 466, 4083-4093 (2017). 
36. Briggs, D. S. High Fidelity Interferometric Imaging: Robust Weighting and NNLS Deconvolution. In American Astronomical Society Meeting Abstracts, vol. 27 of Bulletin of the American Astronomical Society, 1444 (1995).

37. Draine, B. T. et al. Dust Masses, PAH Abundances, and Starlight Intensities in the SINGS Galaxy Sample. ApJ 663, 866-894 (2007).

38. Hueso, R. \& Guillot, T. Evolution of protoplanetary disks: constraints from DM Tauri and GM Aurigae. A\&A 442, 703-725 (2005).

39. Robitaille, T. P., Whitney, B. A., Indebetouw, R., Wood, K. \& Denzmore, P. Interpreting Spectral Energy Distributions from Young Stellar Objects. I. A Grid of 200,000 YSO Model SEDs. ApJS 167, 256-285 (2006).

40. Takakuwa, S. et al. A Keplerian Circumbinary Disk around the Protostellar System L1551 NE. ApJ 754, 52 (2012).

41. Andrews, S. M. et al. Resolved Images of Large Cavities in Protoplanetary Transition Disks. ApJ 732, 42 (2011).

42. Lee, C.-F. et al. SiO Shocks of the Protostellar Jet HH 212: A Search for Jet Rotation. ApJ 685, 1026-1032 (2008).

43. Jørgensen, J. K. et al. PROSAC: a submillimeter array survey of low-mass protostars. II. The mass evolution of envelopes, disks, and stars from the Class 0 through I stages. $A \& A \mathbf{5 0 7}$, 861-879 (2009). 
44. Kwon, W., Looney, L. W., Mundy, L. G., Chiang, H.-F. \& Kemball, A. J. Grain Growth and Density Distribution of the Youngest Protostellar Systems. ApJ 696, 841-852 (2009).

45. Chiang, H.-F., Looney, L. W. \& Tobin, J. J. The Envelope and Embedded Disk around the Class 0 Protostar L1157-mm: Dual-wavelength Interferometric Observations and Modeling. ApJ 756, 168 (2012).

46. Tobin, J. J. et al. Modeling the Resolved Disk around the Class 0 Protostar L1527. ApJ 771, 48 (2013).

47. Miotello, A. et al. Grain growth in the envelopes and disks of Class I protostars. A\&A 567, A32 (2014).

48. Lee, C.-F. et al. First detection of equatorial dark dust lane in a protostellar disk at submillimeter wavelength. Science Advances 3, e1602935 (2017).

49. Bohren, C. F. \& Huffman, D. R. Absorption and scattering of light by small particles (New York: Wiley, 1983, 1983).

50. Weingartner, J. C. \& Draine, B. T. Dust Grain-Size Distributions and Extinction in the Milky Way, Large Magellanic Cloud, and Small Magellanic Cloud. ApJ 548, 296-309 (2001).

51. Draine, B. T. Scattering by Interstellar Dust Grains. I. Optical and Ultraviolet. ApJ 598, $1017-1025$ (2003).

52. Mathis, J. S., Rumpl, W. \& Nordsieck, K. H. The size distribution of interstellar grains. ApJ 217, 425-433 (1977). 
53. Draine, B. T. On the Submillimeter Opacity of Protoplanetary Disks. ApJ 636, 1114-1120 (2006).

54. Wilson, T. L. \& Rood, R. Abundances in the Interstellar Medium. ARA\&A 32, 191-226 (1994).

55. Harsono, D., Visser, R., Bruderer, S., van Dishoeck, E. F. \& Kristensen, L. E. Evolution of CO lines in time-dependent models of protostellar disk formation. A\&A 555, A45 (2013).

56. Jones, E., Oliphant, T., Peterson, P. et al. SciPy: Open source scientific tools for Python (2001-). URL http: //www.scipy • org/. [Online; accessed ¡todayi].

57. Foreman-Mackey, D., Hogg, D. W., Lang, D. \& Goodman, J. emcee: The MCMC Hammer. PASP 125, 306-312 (2013).

58. Murillo, N. M., Lai, S.-P., Bruderer, S., Harsono, D. \& van Dishoeck, E. F. A Keplerian disk around a Class 0 source: ALMA observations of VLA1623A. A\&A 560, A103 (2013).

59. White, R. J. \& Hillenbrand, L. A. On the Evolutionary Status of Class I Stars and Herbig-Haro Energy Sources in Taurus-Auriga. ApJ 616, 998-1032 (2004).

60. Ansdell, M. et al. ALMA Survey of Lupus Protoplanetary Disks. I. Dust and Gas Masses. ApJ 828, 46 (2016).

61. Kama, M. et al. Observations and modelling of $\mathrm{CO}$ and [C i] in protoplanetary disks. First detections of [C i] and constraints on the carbon abundance. A\&A 588, A108 (2016). 
62. Guilloteau, S. et al. Chemistry in disks. X. The molecular content of protoplanetary disks in Taurus. $A \& A$ 592, A124 (2016).

63. Miotello, A. et al. Lupus disks with faint CO isotopologues: low gas/dust or high carbon depletion? A\&A 599, A113 (2017).

64. Schöier, F. L., van der Tak, F. F. S., van Dishoeck, E. F. \& Black, J. H. An atomic and molecular database for analysis of submillimetre line observations. A\&A 432, 369-379 (2005).

65. Yang, B., Stancil, P. C., Balakrishnan, N. \& Forrey, R. C. Rotational Quenching of CO due to $\mathrm{H}_{2}$ Collisions. ApJ 718, 1062-1069 (2010).

66. Neufeld, D. A. Collisional Excitation of Far-infrared Line Emissions from Warm Interstellar Carbon Monoxide (CO). ApJ 749, 125 (2012).

67. Harsono, D., Bruderer, S. \& van Dishoeck, E. F. Volatile snowlines in embedded disks around low-mass protostars. $A \& A$ 582, A41 (2015).

68. Kristensen, L. E. et al. Water in star-forming regions with Herschel (WISH). II. Evolution of $557 \mathrm{GHz} 1_{10}-1_{01}$ emission in low-mass protostars. A\&A 542, A8 (2012).

69. Yvart, W., Cabrit, S., Pineau des Forêts, G. \& Ferreira, J. Molecule survival in magnetized protostellar disk winds. II. Predicted $\mathrm{H}_{2} \mathrm{O}$ line profiles versus Herschel/HIFI observations. $A \& A$ 585, A74 (2016). 
70. van Dishoeck, E. F. et al. Water in Star-forming Regions with the Herschel Space Observatory (WISH). I. Overview of Key Program and First Results. Publications of the Astronomical Society of the Pacific 123, 138-170 (2011).

71. Kratter, K. \& Lodato, G. Gravitational Instabilities in Circumstellar Disks. ARA\&A 54, 271311 (2016).

72. Lodato, G. \& Rice, W. K. M. Testing the locality of transport in self-gravitating accretion discs - II. The massive disc case. MNRAS 358, 1489-1500 (2005).

73. Cossins, P., Lodato, G. \& Clarke, C. J. Characterizing the gravitational instability in cooling accretion discs. MNRAS 393, 1157-1173 (2009).

74. Shakura, N. I. \& Sunyaev, R. A. Black holes in binary systems. Observational appearance. $A \& A$ 24, 337-355 (1973).

75. Kratter, K. M., Matzner, C. D., Krumholz, M. R. \& Klein, R. I. On the Role of Disks in the Formation of Stellar Systems: A Numerical Parameter Study of Rapid Accretion. ApJ 708, $1585-1597$ (2010).

76. Bruderer, S. Survival of molecular gas in cavities of transition disks. I. CO. A\&A 559, A46 (2013).

77. Visser, R., van Dishoeck, E. F. \& Black, J. H. The photodissociation and chemistry of CO isotopologues: applications to interstellar clouds and circumstellar disks. A\&A 503, 323-343 (2009). 0906.3699 . 
78. van Dishoeck, E. F., Jonkheid, B. \& van Hemert, M. C. Photoprocesses in protoplanetary disks. Faraday Discussions 133, 231-243 (2006). 